\title{
AVALIAÇÃO DE GENÓTIPOS DOS CAFEEIROS ARABICA E ROBUSTA NA AMAZÔNIA OCIDENTAL
}

\author{
Rita de Cassia A. PEREIRA*, Francisco J. da S. LÉDO*, Celso L. BERGO*, \\ Amauri SIVIERO*
}

\begin{abstract}
RESUMO - Avaliou-se o comportamento de 16 genótipos de cafeeiros das espécies $C$, arabica e C. canephora nas condições edafoclimáticas do estado do Acre. Os genótipos utilizados foram provenientes do extinto Instituto Brasileiro do Café (Natividade, RJ). O experimento foi conduzido no campo experimental da Embrapa Acre, Rio Branco, AC, no período de 1989 a 1996, num delineamento experimental de blocos casualizados, com 5 repetições. As características estudadas foram: produção de café em cereja e côco, vigor, altura da planta, diâmetro do caule e aspectos fitossanitários. Os genótipos Icatu PR182039-1 e Conilon ES foram os que apresentaram as maiores produções médias de café côco, 4.345 e $4.147 \mathrm{~kg} / \mathrm{ha}$, respectivamente, e em seguida vieram o Catuai SH1 EP57C-260, Mundo Novo e Catuai SH1 EP57C-166, todos com produção acima de $2.800 \mathrm{~kg} / \mathrm{ha}$. Quanto a incidência de ferrugem (Hemileia vastratrix), os genótipos Mundo Novo e Catuaí Amarelo apresentaram maior incidencia, e os mais susceptiveis a queima do fio (Pellicularia koleroga) foram Catuai EP 57-C-260 e Catuai EP57 C-166. Os genótipos Icatu PR182039-1, Conilon ES e Catuai SH1 EP57C-260 foram superiores aos demais genótipos quanto a capacidade produtiva, bom aspecto dos frutos e grãos, mostrando-se promissores para o cultivo no Estado do Acre.
\end{abstract}

Palavras-chave: café, Coffea canephora, Coffea arabica, produtividade, Hemileia vastratrix, Pellicularia koleroga

\section{Evaluation of Arabica and Robusta Coffee Genotypes in Western Amazonia}

ABSTRACT - The behavior of 16 genotypes of coffee ( $C$. arabica and $C$. canephora) was evaluated in the edaphoclimatic conditions of the state of Acre. The genotypes came from the extinct Brazilian Coffee Institute (Natividade, RJ). The experiment was conducted at Embrapa Acre, Rio Branco, AC, in the period from 1989 to 1996, in a randomized block design, with five repetitions. The characteristics studied were yield of "cherry" fruits and coffee beans, vigor, total height, stem diameter and phytossanitary conditions. The Icatu PR182039-1 and Conilon ES genotypes showed higher average yield of beans, with 4.345 and $4.147 \mathrm{~kg} / \mathrm{ha}$, respectively, followed by Catuai SH1 EP57C-260, Mundo Novo and Catuai SH1 EP57C-166, with yields above $2.800 \mathrm{~kg} / \mathrm{ha}$. Mundo Novo and Catuai Amarelo presented higher incidence of Hemileia vastratrix, and Catuai EP 57-C-260 and Catuai EP57 C-166 were more susceptible to Pellicularia koleroga. Icatu PR182039-1, Conilon ES and Catuai SH1 EP57C-260 were superior to the other genotypes with respect to plant vigor, yield capacity, appearance of the cherries and beans, and show promise for cultivation in the state of Acre.

Key Words: coffee, Coffea canephora, Coffea arabica, yield, Hemileia vastratrix, Pellicularia koleroga

\section{INTRODUÇÃO}

A cultura do café no estado do Acre vem tornando-se uma boa opção para o pequeno produtor, tendo em vista a sua potencialidade na geração de receita, constituindo-se em um importante fator de fixação do homem ao campo. Seu cultivo ocorre em quase todos os municípios acreanos, em altitudes que variam de 100 a 600 metros, com temperatura média anual

*Embrapa Acre, Cx. Postal 392, 69.901-180 Rio Branco, AC. 
em torno de $24,5^{\circ} \mathrm{C}$, pluviosidade média anual acima de $2000 \mathrm{~mm}$ e umidade relativa do ar em torno de 80 95\% (Embrapa, 1995). Estima-se atualmente o parque cafeeiro do Estado em 3 milhões de pés, sendo $60 \%$ de café robusta e $40 \%$ de café arábica, com um rendimento médio de $974 \mathrm{~kg} / \mathrm{ha}$ de café em côco (IBGE, 1994).

As cultivares de café utilizadas pelos produtores do Acre foram introduzidas sem nenhum critério técnico. Assim, muito dos materiais utilizados não apresentam boa adaptação para as condições locais, o que contribuiu para a baixa produtividade observada no Estado. $\mathrm{O}$ surgimento de novas cultivares de café de porte baixo, rústicas e de alta produtividade, como o Catuai Vermelho e Catuaí Amarelo, desenvolvidas pelo Instituto Agronômico de Campinas, promoveram uma mudança no sistema de produção tradicional, com aumento da lucratividade, viabilizando a utilização de novas áreas (Fazuoli et al., 1996). Recentemente, com a introdução da cultivar Icatu nas diferentes regiões cafeeiras do país, torna-se necessário avaliar o comportamento vegetativo-produtivo dessas novas cultivares para as diferentes condições edafoclimáticas do Brasil. (Sertorio et al., 1996),

Neste contexto, a Embrapa Acre vem desenvolvendo trabalhos de introdução, avaliação e caracterização de genótipos de cafeeiros, com vistas a identificar genótipos adaptados às condições edafoclimáticas do Acre, com alto potencial produtivo, resistência e ou tolerância às principais pragas e doenças, e grãos com bom aspecto comercial.

\section{MATERIAL E MÉTODOS}

Foram avaliados 15 genótipos de cafeeiros da espécie $C$. arábica e 1 da espécie $C$. canephora (Tab. 1). O experimento foi conduzido no período de janeiro de 1989 a maio de 1996, no campo experimental da Embrapa Acre, em Rio Branco, AC, num solo Podzólico Vermelho-Amarelo, anteriormente coberto por floresta primária, que foi brocada, derrubada e queimada. As mudas foram transplantadas para o local definitivo no espaçamento de $3,0 \mathrm{~m}$ entre fileiras e 2,0 m entre plantas na fileira, com duas plantas por cova. No plantio e durante a condução do experimento foram realizadas adubações e tratos culturais conforme as recomendações comumente utilizadas para a cultura (Pereira et al., 1996).

Os tratamentos foram dispostos no delineamento de blocos casualizados com 5 repetições, sendo cada parcela constituida por 6 plantas (2 plantas por cova). Utilizou-se como bordadura geral a linhagem Icatu-a.

Foram avaliadas as seguintes características:

\section{Produção de Café Cereja e Côco}

As colheitas foram efetuadas quando aproximadamente $80 \%$ dos frutos estavam maduros (cereja), nos meses de março a maio, no período de 1991-96 (seis safras), em intervalos de 4 a 5 dias. A produção da parcela foi 
pesada, obtendo-se a produção de café cereja, e em seguida submetida a secagem e beneficiamento, para avaliação da produção de café côco.

\section{Aspecto Vegetativo}

Foi determinado com base no vigor, altura e diâmetro do caule das plantas. $\mathrm{O}$ vigor foi avaliado de modo subjetivo a cada ano antes da colheita, atribuindo-se nota de 1 a 10 , sendo a nota 1 correspondente às piores plantas e 10 aos cafeeiros com melhor desenvolvimento. A altura foi determinada a partir do nivel do solo e o diâmetro do caule foi medido a 10 $\mathrm{cm}$ do solo: ambas as características foram avaliadas apenas no ano de 1995.

\section{Aspectos Fitossanitários}

Para avaliar a resistência à ferrugem (Hemileia vastatrix), seguiuse a metodologia adaptada por Siviero et al. (1996): avaliou-se 24 plantas de cada genótipo, coletando-se 4 folhas por planta, sendo duas folhas entre as fileiras e duas folhas dentro das

Tabela 1. Identificação e origem dos genótipos de cafeeiros avaliados em Rio Branco, AC, 1996.

\begin{tabular}{lll}
\hline Genótipo & Código original & Origem \\
\hline lcatu-a & PR. 1.82039-1 (H 4782-16) & Londrina/PR \\
Icatu-b & PR. 1.75024-6-1 (H 4782-10) & Londrina/PR \\
lcatu-c & PR. 1.82038-1 (H 4782-16) & Londrina/PR \\
lcatu-d & PR. 1.75006-6-2-1 (H 4782-13-79) & Londrina/PR \\
Icatu-e & PR. 1.75024-5-13 (H 4782-10) & Londrina/PR \\
Catuaí SH1-a & EP 57 C-260 & Natividade/RJ \\
Catuai SH1-b & EP 57 C-166 & Natividade/RJ \\
Catuaí Vermelho-a & ENAT 6.C.33 (H 2077-2-5-114 e.444) & Natividade/RJ \\
Catuaí Vermelho-b & ENAT 6.C.13 (H 2077-2-5-114 e.444) & Natividade/RJ \\
Catuaí Vermelho-c & ENAT 6.C.34 (H 2077-2-5-114 e.444) & Natividade/RJ \\
Catuai SH2 SH3 & ENAT 6.C.60 (H 6839-5) & Natividade/RJ \\
Catuaí Amarelo & ENAT 6.C.3 (LCH 2077-2-5-62) & Natividade/RJ \\
Icatu x Catimor & ENAT 7.C.35 (H PR. 77026-01) & Natividade/RJ \\
C. Vermelho $\times$ Catimor & ENAT 7.C.44 (H PR. 77012-12) & Natividade/RJ \\
Conilon ES & Linh. Campo Viveiro Conilon ES & Natividade/RJ \\
Mundo Novo & PR. 1.L 88001 (LCP 388-17) & Londrina/RJ \\
\hline
\end{tabular}


fileiras. A intensidade da doença (severidade) foi medida pelo uso de uma escala de notas, atribuindo-se 1 (ausência de pústulas) à 8 (pústulas uredospóricas grandes cloróticas e/ou necróticas). A média destas avaliações serviu como um índice de incidência da doença para cada genótipo. A ocorrência de queima do fio (Pellicularia koleroga) foi avaliada em março de 1996, usando uma escala de notas variando de 0 a 3 , onde 0 : ausência de sintomas, $1: 1$ a 2 focos pequenos da doença, 2: mais de 2 focos pequenos da doença e 3: mais de 2 focos grandes da doença. Cada planta recebeu nota individual e a média foi transformada em $\sqrt{x+0,5}$.

\section{RESULTADOS E DISCUSSÃO}

A maioria dos genótipos avaliados iniciou sua produção (Tab. 2) a partir de 1991, destacando-se neste ano, os genótipos Catuai SH1-a,

Tabela 2. Produção de café côco em 16 genótipos de cafeeiros, avaliados no periodo de 19911996 em Rio Branco, AC.

\begin{tabular}{lrrrrrrr}
\hline & \multicolumn{7}{c}{ Produção de café côco (kgha) } \\
\cline { 2 - 8 } Genótipos & 1991 & 1992 & 1993 & 1994 & 1995 & 1996 & Média* $^{*}$ \\
\hline Icatu-a & 1.783 & 1.039 & 6.792 & 5.422 & 7.680 & 3.167 & $4.345 \mathrm{a}$ \\
Icatu-b & 968 & 906 & 3.558 & 3.900 & 2.344 & 3.778 & $2.575 \mathrm{c}$ \\
Icatu-c & 1.267 & 237 & 1.859 & 1.879 & 1.578 & 4.767 & $1.930 \mathrm{c}$ \\
lcaut-d & 979 & 424 & 1.752 & 2.800 & 2.175 & 2.067 & $1.700 \mathrm{c}$ \\
Icatu-e & 1.011 & 441 & 1.000 & 1.611 & 674 & 3.333 & $1.345 \mathrm{c}$ \\
Catuaí SH1-a & 2.966 & 1.680 & 3.548 & 3.013 & 1.867 & 5.111 & $3.031 \mathrm{~b}$ \\
Catuaí SH1-b & 2.480 & 917 & 3.376 & 2.367 & 2.344 & 5.711 & $2.865 \mathrm{~b}$ \\
Catuai Vermelho-a & 1.670 & 1.378 & 3.458 & 2.839 & 1.789 & 4.211 & $2.443 \mathrm{c}$ \\
Catuaí Vermelho-b & 1.706 & 1.022 & 1.546 & 2.322 & 1.222 & 4.089 & $1.977 \mathrm{c}$ \\
Catuaí Vermelho-c & 629 & 652 & 1.505 & 1.779 & 709 & 2.200 & $1.255 \mathrm{c}$ \\
Catuaí SH2 SH3 & 1.410 & 611 & 1.916 & 1.933 & 1.039 & 2.855 & $1.627 \mathrm{c}$ \\
Catuaí Amarelo & 1.956 & 1.024 & 1.728 & 2.511 & 1.305 & 3.033 & $1.926 \mathrm{c}$ \\
lcatu x Catimor & 612 & 794 & 1.838 & 2.379 & 1.955 & 2.289 & $1.586 \mathrm{c}$ \\
C. Verm.x Catimor & 896 & 579 & 1.649 & 2.179 & 955 & 2.811 & $1.493 \mathrm{c}$ \\
Conilon ES & 2.044 & 2.940 & 5.189 & 5.600 & 3.489 & 5.622 & $4.147 \mathrm{a}$ \\
Mundo Novo & 1.606 & 1.272 & 4.576 & 3.156 & 3.469 & 3.689 & $2.961 \mathrm{~b}$ \\
\hline
\end{tabular}

"Médias seguidas de uma mesma letra năo diferem entre si, em nível de $5 \%$ de probabilidade, pelo teste Scott-Knott (1974). 
Catuai SH1-b e Conilon ES. Em geral, os melhores picos de produção foram registrados a partir do terceiro ano de colheita (1993), coincidindo com Fazuoli (1977), que avaliou progênies de café Mundo Novo.

Com exceção do vigor, todos os caracteres agronômicos avaliados apresentaram efeito significativo $(\mathrm{P}<$ $0,05)$. Os genótipos Icatu-a e Conilon ES foram os que apresentaram as maiores produções de café cereja (10.704 e $9.693 \mathrm{~kg} / \mathrm{ha})$ e côco (4.345 e $4.147 \mathrm{~kg} / \mathrm{ha}$, respectivamente), enquanto que o Catuai SH1-a, Mundo Novo e Catuaí SH1-b apresentaram produção de café em côco acima de $2.800 \mathrm{~kg} / \mathrm{ha}$, o que pode ser considerado uma excelente produtividade, tendo em vista a média apresentada pelo estado, que situa-se em torno de $974 \mathrm{~kg} / \mathrm{ha}$ (IBGE, 1994). A seleção de genótipos, com base na produção média verificada a partir da terceira ou quarta colheita, tem mostrado boa confiabilidade, sendo que a eficiência de seleção é razoavelmente alta a partir da quarta colheita (Carvalho et al., 1973; Mendes et al., 1996).

O genótipo Icatu-a apresentou maior altura da planta, $3,4 \mathrm{~m}$, e os materiais $\mathrm{C}$. Vermelho $\mathrm{x}$ Catimor, Catuai Vermelho-c, Icatu x Catimor, Catuaí SH1-a, Catuaí SH2 SH3, Catuaí Vermelho-b, C. Vermelho-a e Catuai Amarelo as menores, variando de 2,3 a 2,6 m (Tab. 3). Alvarenga et al (1995) também constataram que o Catuaí apresentou menor altura quando comparada com progênies de café Icatu. Em geral os genótipos apresentaram altura elevada, que pode ser explicada, em parte, pelas condições climáticas da região, caracterizadas por temperatura e precipitação elevadas e curto período de estiagem (Oliveira, 1980). Com relação ao diâmetro do caule, os genótipos Conilon ES e Icatu-a apresentaram os maiores valores, 8,1 e $8,0 \mathrm{~cm}$.

Quanto aos aspectos fitossanitários, em apenas uma avaliação realizada, observou-se o ataque moderado da 'queima do fio', causada pelo fungo Pelicularia koleroga, em todos os materiais testados, sendo os genótipos Catuaí SH1-a, Catuai SH1-b, Catuaí Vermelho-b e Catuai SH2 SH3 os mais susceptíveis (Tab, 3). O mesmo patógeno já foi observado afetando cafeeiros consorciados com seringueira em Manaus, AM (Silva et al., 1983). No Equador, onde o clima é semelhante ao do experimento, a 'queima do fio' é considerada a principal doença do cafeeiro (Herrera \& Guambi, 1995a; 1995b).

De forma geral, a incidência de ferrugem (Hemileia vastatrix) foi baixa nos genótipos testados. O maior ataque foi observado no Mundo Novo e Catuaí Amarelo; os demais apresentaram baixos níveis (Tab, 3). Resultados semelhantes foram verificados por Carvalho \& Fazuoli (1993), onde o Mundo Novo mostrouse susceptível à ferrugem, apesar da elevada capacidade produtiva. Nossas 
Tabela 3. Produção de café cereja (PCE), altura da planta (AP), diâmetro do caule (DC), vigor, indice de ocorrência de queima do Fio (QF) e ferrugem (F) em 16 genótipos de cafeeirosm em Rio Branco, AC.

\begin{tabular}{|c|c|c|c|c|c|c|}
\hline Genótipos & $\begin{array}{c}\text { PCE (kgha)* } \\
1991-96 \\
\end{array}$ & $\begin{array}{c}\operatorname{AP}(m)^{*} \\
1995\end{array}$ & $\begin{array}{c}\mathrm{DC}(\mathrm{cm})^{*} \\
1995\end{array}$ & $\begin{array}{l}\text { Vigor } \\
91-96 \\
\end{array}$ & $\begin{array}{l}\mathrm{QF}^{*} \\
1996 \\
\end{array}$ & $\begin{array}{c}F^{*} \\
1996 \\
\end{array}$ \\
\hline Icatu-a & $10.704 \mathrm{a}$ & $3,4 \mathrm{a}$ & $8,0 \mathrm{a}$ & 6,6 & $0,46 b$ & $1,03 \mathrm{~b}$ \\
\hline Icatu-b & 7.357 a & $3,0 \mathrm{~b}$ & $6,3 \mathrm{~b}$ & 5,5 & $0,93 \mathrm{~b}$ & $1,03 \mathrm{~b}$ \\
\hline Icatu-c & $4.962 \mathrm{~b}$ & $2,8 \mathrm{~b}$ & $5,8 \mathrm{c}$ & 5,4 & $0,86 \mathrm{~b}$ & $1,03 \mathrm{~b}$ \\
\hline Icatu-d & $3.933 \mathrm{~b}$ & $2,8 b$ & $6,6 \mathrm{~b}$ & 5,8 & $0,93 b$ & $1,00 \mathrm{~b}$ \\
\hline Icatu-e & $3.173 b$ & $2,7 b$ & $5,1 \mathrm{C}$ & 4,7 & $0,33 \mathrm{~b}$ & $1,20 \mathrm{~b}$ \\
\hline Catuai SH1-a & $7.816 \mathrm{a}$ & $2,3 \mathrm{c}$ & $5,4 \mathrm{c}$ & 6,1 & $2,20 \mathrm{a}$ & $1,05 \mathrm{~b}$ \\
\hline Catuai SH1-b & $6.938 \mathrm{a}$ & $2,3 \mathrm{c}$ & $4,9 \mathrm{c}$ & 6,2 & $1,46 a$ & $1,25 b$ \\
\hline Catuaí Vermelho-a & $5.735 b$ & $2,4 \mathrm{C}$ & $4,5 \mathrm{c}$ & 5,3 & $0,86 \mathrm{~b}$ & $1,55 \mathrm{~b}$ \\
\hline Catuai Vermelho-b & $4.309 \mathrm{~b}$ & $2,4 \mathrm{C}$ & $4,7 \mathrm{c}$ & 5,2 & $1,40 \mathrm{a}$ & $1,38 \mathrm{~b}$ \\
\hline Catuai Vermelho-c & $2.751 \mathrm{~b}$ & $2,3 \mathrm{c}$ & $4,7 \mathrm{c}$ & 5,3 & $0,86 \mathrm{~b}$ & $1,43 b$ \\
\hline Catuai SH2 SH3 & $3.409 \mathrm{~b}$ & $2,4 \mathrm{c}$ & $4,7 \mathrm{c}$ & 5,8 & $1,33 \mathrm{a}$ & $1,08 b$ \\
\hline Catuaí Amarelo & $4.341 \mathrm{~b}$ & $2,6 \mathrm{c}$ & $4,3 \mathrm{c}$ & 5,2 & $0,53 b$ & $2,28 \mathrm{a}$ \\
\hline Icatu x Catimor & $4.273 \mathrm{~b}$ & $2,3 \mathrm{c}$ & $4,5 \mathrm{c}$ & 5,6 & $0,86 \mathrm{~b}$ & $1,05 b$ \\
\hline C. Verm. x Catimor & $3.545 \mathrm{~b}$ & $2,3 \mathrm{c}$ & $4,9 \mathrm{c}$ & 5,7 & $0,73 b$ & $1,08 \mathrm{~b}$ \\
\hline Conilon ES & $9.693 \mathrm{a}$ & $2,8 b$ & $8,1 \mathrm{a}$ & 6,6 & $0,33 b$ & $1,43 b$ \\
\hline Mundo Novo & $7.773 \mathrm{a}$ & $2,9 b$ & $5,8 \mathrm{c}$ & 5,4 & $0,26 \mathrm{~b}$ & $2,53 \mathrm{a}$ \\
\hline
\end{tabular}

*Médias seguidas de uma mesma letra năo diferem entre si, em nivel de $5 \%$ de probabilidade, pelo teste Scott-Knott (1974).

observações em apenas uma avaliação no ano de 1996, confirmaram esta susceptibilidade. Entretanto, não se pode inferir sobre o comportamento destes genótipos quanto a susceptibilidade à ferrugem e a queima do fio nas condições do ambiente testado.

\section{CONCLUSÕES}

Os genótipos Icatu PR1.82039-1, Conilon ES e Catuai SH1 EP57 C-260 apresentaram boa adaptação e produção nas condições do experimento, mostrando-se promissores para o cultivo no Estado do Acre. O Catuai SH1 EP57 C-260, Catuai SH1 EP57 C-160, Catuai Vermelho ENAT 6.C.13 (H 2077-2-5-114 e 444) e Catuai $\mathrm{SH} 2 \mathrm{SH} 3$ mostraram-se susceptiveis a queima do fio. Quanto a incidência de ferrugem, o Mundo Novo e Catuaí Amarelo foram os mais susceptiveis.

\section{Bibliografia citada}

Alvarenga, A. de P.; Begazo, J.C.E.O.; Cardoso, A.A.; Paula, J.F. 1995. Avaliação de progênies de café Icatu, em Viçosa, Minas Gerais. Revista Ceres, 42(243):516- 
527.

Carvalho, A.; Fazuoli, L.C.; Alves, S. 1993. Café. In: Furlani, A.M.C.; Viegas, G.P. (Eds.). O melhoramento de plantas no Instituto Agronómico. Vol.1. Instituto Agronômico, Campinas. p.29-76.

Embrapa. 1995. Boletim agrometeorológico. Vol.1. Embrapa-CPAF/AC, Rio Branco.

Fazuoli, L.C. 1977. Avaliação de progênies de café Mundo Novo (Coffea arabica L.). Dissertação de mestrado, ESALQ, Piracicaba. 146p.

Fazuoli, L.C.; Medina Filho, H.P.; Guerreiro Filho, O.; De Lima, M.M.A.; Silvarolla, M.B. 1996. Cultivares e linhagens de café lançadas pelo Instituto Agronômico de Campinas (IAC). In: Congresso brasileiro de pesquisas cafeeiras, 22, 1996, Águas de Lindóia, SP. Anais... MAARA/ PROCAFÉ, Águas de Lindóia. p.147.

Herrera, 1.S.; Guambi, L.D. 1995a. Control integrado de las principales enfermidades foliares del cafeto en el Ecuador. INIAPGTZ, Pinchilingue. 78p.

Herrera, I.S.; Guambi, L.D. 1995b. Inventário tecnológico del cultivo de café. INIAPGTZ, Pinchilingue, 103p.

IBGE. 1994. Produçào Agricola Municipal: Acre. Vol.1. Inst. Bras, Geog. e Estat., Rio Branco, 50p.

Mendes, A.N.G.; Ramalho, M.A.P.; Pereira, A.A.; Bártholo, G.F. 1996. Métodos de avaliação de progênies de Coffea arabica L., cultivar Catuaí. Ciência e Agrotecnologia, 20(3):315-322.
Oliveira, V.H. 1980. Aspectos gerais sobre a cultura do café no Acre. (Circular Técnica, 2) EMBRAPA-UEPAE, Rio Branco. 20p.

Pereira, R. de C.A.; Sá, C.P. de; Sales, F. de. 1996. Recomendações básicas para a cultura do cafeeiro no estado do Acre. (Circular Técnica, 14) Embrapa-CPAF/ AC, Rio Branco, 20p.

Scott, A.J.; Knott, M. 1974. A cluster analysis method for grouping means in the analyses of variance. Biometrics, 30:507-512.

Sertório, R.; Santinato, R.; Silva, V.A.; Carvalho, R. 1996. Comportamento do Icatu 2945 em competição com as variedades comerciais Catuai e Mundo Novo nas condiçõ̀es de Pinhal - SP Fase de formação. In: Congresso brasileiro de pesquisas cafeeiras, 22, 1996. Águas de Lindóia, SP. Anais... MAARA/ PROCAFÉ, Ȧguas de Lindóia. p.142.

Silva, H.M.; Gasparotto, L.; Trindade, R. 1983. Pellicularia koleroga em seringais consorciados na Amazônia. Fitopatologia Brasileira, 8(3):601.

Siviero, A.; Pereira, R. de C.A.; Sales, F. de.; Sampaio, F.R.V. 1996. Reação preliminar de linhagens de cafeeiros (Coffea arabica) a Hemileia vastratrix no Estado do Acre. Fitopatologia Brasileira, 21(Supl.):384. 